\title{
Magnetic moment of Eu-dopant and superconductivity in Eu-substitutional $\mathrm{Ba}_{8} \mathrm{Al}_{5} \mathrm{Si}_{41}$ clathrates
}

Lihua Liu ${ }^{\mathrm{a}}$, Yueting Ren ${ }^{\mathrm{a}}$, Bensheng Song ${ }^{\mathrm{a}}$, Jose Garcia ${ }^{\mathrm{b}}$, Hengyi Du ${ }^{\mathrm{b}}$, Xingqiao $\mathrm{Ma}^{\mathrm{a}}$, Yang Li ${ }^{\mathrm{b}}$

${ }^{a}$ Department of Physics, University of Science and Technology Beijing, Beijing 100083, China

${ }^{\mathrm{b}}$ Department of Engineering Science and Materials, University of Puerto Rico at Mayaguez, Mayaguez, Puerto Rico 00681-9000, USA

\begin{abstract}
Si clathrates $\mathrm{Ba}_{8-x} \mathrm{Eu}_{x} \mathrm{Al}_{5} \mathrm{Si}_{41}$ with nominal $x=0,0.1,0.2,0.3$ and 0.4 have been synthesized by arc melting and acid washing so as to delete impure phases. Eu substitution for $\mathrm{Ba}$ in the clathrate results in introduction of magnetic moment and modification of crystalline structure. The decrease of the cubic lattice parameter $a$ with the Eu content implies the incorporation of the Eu into the cages. The critical superconducting transition temperature $T_{\mathrm{C}}$ decreases from 6.8 to $4.5 \mathrm{~K}$ as $x$ increases from 0 to 0.3 . For sample with nominal $x=0.4$, no diamagnetic behavior indicative of superconductivity was found down to $2 \mathrm{~K}$. These suggest that due to the introduction of the magnetic moment of $\mathrm{Eu}$, the superconductivity was suppressed. In addition, the ferromagnetic interaction was also observed at the same time. For the Eu-free sample, both $M-T$ and $M-H$ curves show the typical behavior of type-II superconductivity. With the increases of $\mathrm{Eu}$ content, the superconducting lower critical field $H_{\mathrm{C} 1}$ decreases and Weiss temperature increases. As $x$ reaches to 0.4 , superconductivity is totally suppressed and the sample shows only ferromagnetic behavior at low temperature.
\end{abstract}

Key Words: type-I Si clathrate, Eu-substitution, superconductivity.

\section{Introduction}

Group IV clathrates have obtained great attention since mid-1990s in the fields including thermoelectricity [1-4], superconductivity [5-8], wide bandgap semiconductivity [9], and mechanical properties [10], etc. For all these properties, superconductivity is probably the most attractive one because the clathrate structure is dominated by strong covalent bonds, rather than the more typical metallic bonds found in traditional superconductors.

The fully Ba-caged $\mathrm{Ba}_{8} \mathrm{Si}_{46}$ compound is the clathrate which shows the highest superconductivity transition temperature $T_{\mathrm{C}}$ at $8 \mathrm{~K}$. The framework of $\mathrm{Ba}_{8} \mathrm{Si}_{46}$ is composed of $\mathrm{Si}_{20}$ and $\mathrm{Si}_{24}$ cages formed with $s p^{3}$-hybridized $\mathrm{Si}$-Si covalent bonds. Guest atoms (Ba) are endohedrally intercalated in the cages. Many research groups are engaged in the research and development of this superconducting material. Any substitutions of $\mathrm{Si}$ on the framework or Ba in the center of the cage can profoundly affect superconductivity [11-12]. The destructive effect on 
superconductivity generally comes from several reasons: (i) the decrease in density of state at Fermi-level $\left(N\left(E_{\mathrm{F}}\right)\right)$, (ii) a lowering of the carrier concentration, (iii) a breakage of the integrity of the $s p^{3}$-hybridized network, (iv) the magnetic breakdown of paired electrons, and (v) the red shift of phonon dispersion spectra, which resulting in the depression of electron-phonon coupling.

In our study of clathrates, a new method to synthesize pure Ba-Si type-I clathrates have been developed in the conventional equipment with arc melting but not the high-pressure-high-temperature method [13]. A series of $\mathrm{Al}$ substituted $\mathrm{Ba}_{8} \mathrm{Si}_{46-x} \mathrm{Al}_{x}$ single phase samples were prepared by using this method. Then we prepared $\mathrm{Eu}$ - and $\mathrm{Al}$-doped $\mathrm{Ba}-\mathrm{Si}$ clathrates. The influence of large magnetic moment of Eu on superconductivity for $\mathrm{Ba}_{8-x} \mathrm{Eu}_{x} \mathrm{Al}_{6} \mathrm{Si}_{40}$ ( $x=0$, $0.5,1$, and 2 ) has been discussed in ref [14]. The incorporation of Eu strongly destroys superconductivity as a result of magnetic breakdown of pair electrons. However, there has not been the detailed research on superconductivity of dilute Eu-doped clathrates. The evolution of superconducting behavior with gradual increase of Eu-doping can help to understand the effect of magnetic moment on superconductivity. Therefore, dilute Eu-doped $\mathrm{Ba}_{8-x} \mathrm{Eu}_{x} \mathrm{Al}_{5} \mathrm{Si}_{41}$ samples with $x \leqslant 0.4$ were synthesized in this paper. Eu atoms accompanied with magnetic moment enter the clathrate lattice. The dilute magnetism gives rise to the occurrence of ferromagnetic interaction. Therefore, the obtained Weiss temperature by fitting increases with the increase of Eu content. At the same time, this ferromagnetic interaction can destroy superconductivity.

\section{Experiments}

In order to synthesize the quaternary compounds $\mathrm{Ba}_{8-x} \mathrm{Eu}_{x} \mathrm{Al}_{5} \mathrm{Si}_{41}(x=0,0.1,0.2,0.3$ and 0.4 ), metals of $\mathrm{Ba}(99.99 \%), \mathrm{Eu}(99.99 \%), \mathrm{Al}(99.999 \%)$ and $\mathrm{Si}(99.999 \%)$ were weighed in an atomic ration of $[(8-x) \times 1.05]: x: 5: 41$ and melted in arc melting furnace. An extra amount $(5 \%)$ of $\mathrm{Ba}$ was loaded considering $\mathrm{Ba}$ is more volatile and oxidable than other elements. The obtained ingots contain the $8: 46$ clathrate as the main phase. However, a small amount of secondary phases were also dectected in X-ray powder diffraction diagrams, for example, the remaining diamond $\mathrm{Si}, \mathrm{BaSi}_{2}$ and other unidentified phases. In order to get rid of these impurity phases, the ingots were ground thoroughly and washed with dilute $\mathrm{NaOH}$ solution and $\mathrm{HCl}$ acid. The washed powders were flushed with deionized water repeatedly and dried carefully using an evaporator. The powders thus obtained are revealed to be the pure clathrates.

The superconductive $\mathrm{Ba}_{8} \mathrm{Si}_{46}\left(T_{\mathrm{C}} \sim 8.0 \mathrm{~K}\right)$ is a high-pressure phase which cannot be synthesized by using the traditional arc melting method. This has prevented the comprehensive study on clathrate superconductivity for most of researchers due to their lack of high-temperature-high-pressure synthesis equipment. The Al-doped $\mathrm{Ba}_{8} \mathrm{Al}_{5} \mathrm{Si}_{41}$ clathrate is ambient pressure phase with $T_{\mathrm{C}} \sim 6.5 \mathrm{~K}$. That is to say, it can be prepared at ambient pressure by only using arc melting. However, the experiments showed that a sizeable amount of impurity phases, such as $\mathrm{BaSi}_{2}$ and $\mathrm{Si}$, will inevitably appear during the arc melting process. The core technique in the experiments is alkali and acid washing in order to remove impurity phases completely. This method has no harm on physical properties of clathrates and has been 
verified in many Si-containing systems [15-16].

The X-ray diffraction data were collected at room temperature using a Rigaku UltimaIV diffractometer $\left(\mathrm{Cu} K_{\alpha 1}\right.$ radiation, $\lambda=1.54056 \AA 10^{\circ}<2 \theta<80^{\circ}$, step with $0.01^{\circ}$ of $\left.2 \theta\right)$. Structural refinement of the powder X-ray diffraction data was performed employing the GSAS software package. Magnetization is measured by means of a cryogen-free physical properties measurement system (Cryogenic in London). The chemical compositions were determined from the washed samples by field-emission scanning electron microscopy (FE-SEM, SUPRA-55, Carl ZEISS) combined with energy-dispersive X-ray spectroscopy (EDS, X-Max, Oxford Instruments).

\section{Results and Discussions}

\subsection{Structural and chemical characterizations}

To clarify the structure and purity of the washed samples, all X-ray diffraction data were refined by Rietveld method. Based on the results of the refinement, all the washed samples crystallize in the cubic type-I clathrate structure with space group Pm-3n (No. 223). No impurity phases can be observed.

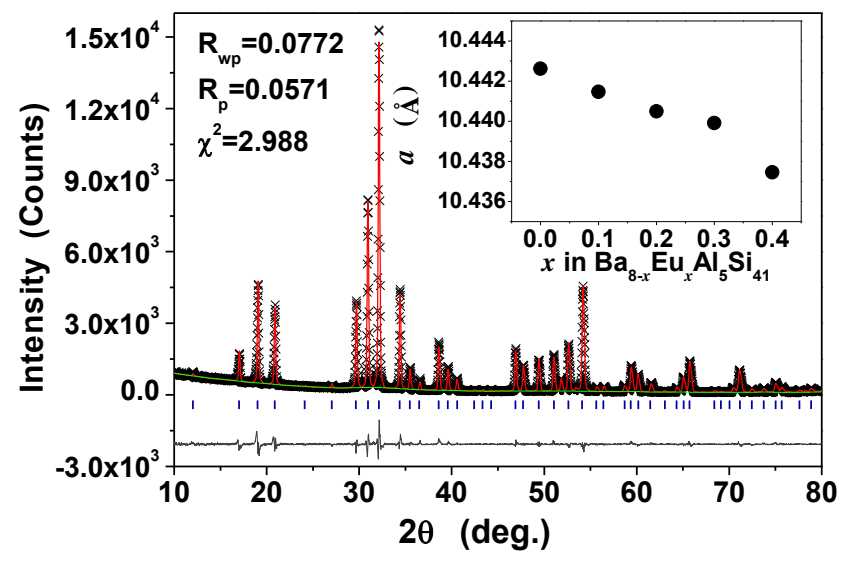

Fig. 1. Refinement of the XRD data of washed $\mathrm{Ba}_{7.7} \mathrm{Eu}_{0.3} \mathrm{Al}_{5} \mathrm{Si}_{41}$ sample (The upper curves: data and fit; the lower curve: the difference plot. Blue ticks show the peaks indexed according to the type-I clathrate structure). Inset: lattice parameters of $\mathrm{Ba}_{8-x} \mathrm{Eu}_{x} \mathrm{Al}_{5} \mathrm{Si}_{41}(x=0,0.1,0.2,0.3$ and 0.4 ), showing a decreasing tread with $x$.

Fig. 1 shows the $\mathrm{X}$-ray refinement result for sample with nominal composition $\mathrm{Ba}_{7.7} \mathrm{Eu}_{0.3} \mathrm{Al}_{5} \mathrm{Si}_{41}$. The experiment pattern is in agreement with the simulated one for the entire $2 \theta$ region. $R$ values for the fit are $R_{\mathrm{wp}}=0.077$ and $R_{\mathrm{p}}=0.052$. The lattice parameter $a$ is $10.44237(9)$, 10.44132(9), 10.44127(9), 10.44005(8) and 10.43764(11) $\AA$ for samples $x=0,0.1,0.2,0.3$ and 0.4 , respectively. As plotted in the inset in Fig. 1, the sizable reduction of the lattice parameter with $x$ implies Eu substitutes for Ba in the cages definitely.

For the clathrate compounds, Europium is the only rare earth element which can be incorporated into the cages, replacing all or sizable amount $\mathrm{Ba}$ atoms without changing the structure. The Eu in the cages is present in divalence and carries a large magnetic moment [17-19]. The Eu atoms were assigned to occupy both the $2 a$ and $6 d$ sites randomly in the refinement model. 
As a result of the refinement, Eu was found to mainly distribute on the $2 a$ sites, with only a few atoms on the $6 d$ sites. This is in accordance with the results of prior studies on Sr-doped $\left(\mathrm{Ba}_{1-\mathrm{x}} \mathrm{Sr}_{\mathrm{x}}\right)_{8} \mathrm{Si}_{46}$ [20]. According to the occupancy of Eu at $2 a$ and $6 d$ sites obtained from refinement, the simulated Eu contents for all samples are shown in Table I. The calculated contents of Eu in clathrates are less than the nominal contents. This might have resulted from the formation of Eu-containing impurity phases which have been removed by alkali and acid washing. On the other hand, $\mathrm{Al}$ atoms were found to mainly occupy the $6 c$ sites, which is in agreement with the previous results, the element substitution on the framework is preferred at $6 c$ sites for various group IV type-I clathrates [21-23].

A scanning electronic microscopy combined with EDS analysis was performed on all samples. The EDS analysis were performed on the large grains of main phase clathrate, and the data were collected from six or seven regions. The EDS spatial resolution is about 1 micrometer. The averaged elemental stoichiometries obtained by this method are summarized in Table I. The measured Eu contents are less than the nominal stoichiometries.

Table I. The compositions obtained from XRD refinement and EDS.

\begin{tabular}{ccc}
\hline Nominal composition & Composition by refinement & Composition by EDS \\
\hline $\mathrm{Ba}_{8} \mathrm{Al}_{5} \mathrm{Si}_{41}$ & $\mathrm{Ba}_{7.81} \mathrm{Al}_{6.44} \mathrm{Si}_{39.53}$ & $\mathrm{Ba}_{8.00} \mathrm{Al}_{6.31} \mathrm{Si}_{39.69}$ \\
$\mathrm{Ba}_{7.9} \mathrm{Eu}_{0.1} \mathrm{Al}_{5} \mathrm{Si}_{41}$ & $\mathrm{Ba}_{7.63} \mathrm{Eu}_{0.08} \mathrm{Al}_{6.00} \mathrm{Si}_{39.83}$ & $\mathrm{Ba}_{7.91} \mathrm{Eu}_{0.09} \mathrm{Al}_{6.21} \mathrm{Si}_{39.79}$ \\
$\mathrm{Ba}_{7.8} \mathrm{Eu}_{0.2} \mathrm{Al}_{5} \mathrm{Si}_{41}$ & $\mathrm{Ba}_{7.69} \mathrm{Eu}_{0.16} \mathrm{Al}_{5.95} \mathrm{Si}_{40.05}$ & $\mathrm{Ba}_{7.77} \mathrm{Eu}_{0.23} \mathrm{Al}_{5.87} \mathrm{Si}_{40.13}$ \\
$\mathrm{Ba}_{7.7} \mathrm{Eu}_{0.3} \mathrm{Al}_{5} \mathrm{Si}_{41}$ & $\mathrm{Ba}_{7.61} \mathrm{Eu}_{0.24} \mathrm{Al}_{5.88} \mathrm{Si}_{40.01}$ & $\mathrm{Ba}_{7.75} \mathrm{Eu}_{0.25} \mathrm{Al}_{5.45} \mathrm{Si}_{40.55}$ \\
$\mathrm{Ba}_{7.6} \mathrm{Eu}_{0.4} \mathrm{Al}_{5} \mathrm{Si}_{41}$ & $\mathrm{Ba}_{7.49} \mathrm{Eu}_{0.35} \mathrm{Al}_{5.89} \mathrm{Si}_{40.09}$ & $\mathrm{Ba}_{7.63} \mathrm{Eu}_{0.37} \mathrm{Al}_{5.59} \mathrm{Si}_{40.41}$ \\
\hline
\end{tabular}

3.2. Magnetic heat $(M-T)$ curves

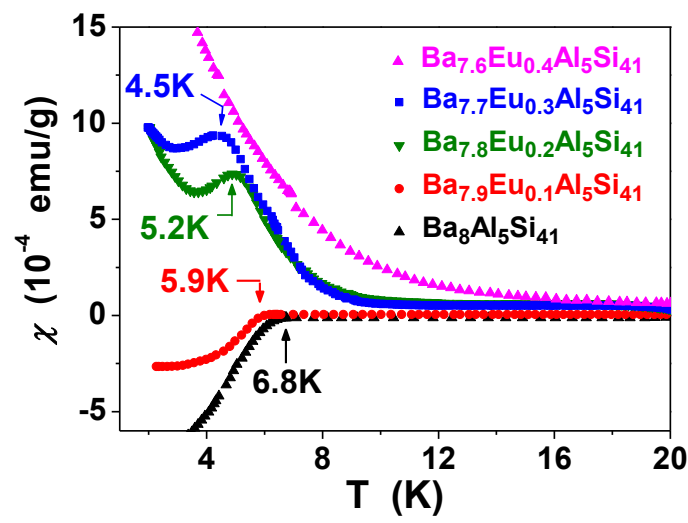

Fig. 2. The temperature dependence of the magnetic susceptibility for specimens $\mathrm{Ba}_{8-x} \mathrm{Eu}_{x} \mathrm{Al}_{5} \mathrm{Si}_{41}(x=0,0.1,0.2,0.3$ and 0.4$)$ under magnetic field $H=$ 100 Oe.

The superconducting critical temperature $T_{\mathrm{C}}$ was determined by dc magnetization on fine powders. Fig. 2 presents the magnetic susceptibility of $\mathrm{Ba}_{8-x} \mathrm{Eu}_{x} \mathrm{Al}_{5} \mathrm{Si}_{41}(x=0,0.1,0.2,0.3$ and 0.4 ) as a function of temperature, under conditions of zero field cooling (ZFC) at 100 Oe. For Eu-free $\mathrm{Ba}_{8} \mathrm{Al}_{5} \mathrm{Si}_{46}, T_{\mathrm{C}}$ is $\sim 6.8 \mathrm{~K}$ and superconducting volume $\left(V_{\text {sup }}\right)$ is $\sim 20.4 \%$. $T_{\mathrm{C}}$ decreases 
with the Eu-doping. No superconducting signal was observed down to $2 \mathrm{~K}$ as $x$ reaches to 0.4 . On the other hand, the Eu substitution also results in a reduction in $V_{\text {sup }}$ as shown in Fig. 2.

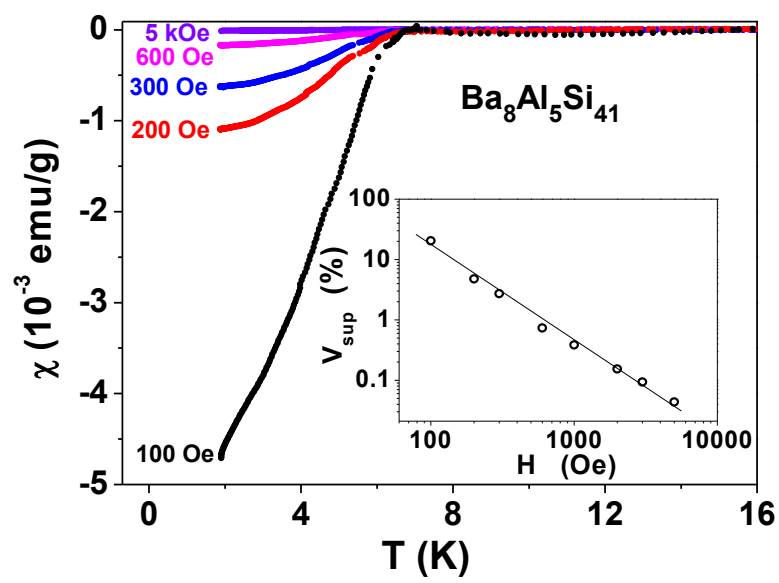

Fig. 3. The ZFC magnetic susceptibility under different field for Eu-free $\mathrm{Ba}_{8} \mathrm{Al}_{5} \mathrm{Si}_{41}$. Inset: Superconducting volume $\left(V_{\text {sup }}\right)$ decreasing with external field according to a linear $\log$ behavior, with the fitted curve $\log V_{\text {sup }}=a+b \log H$.

Fig. 3 shows the ZFC magnetic susceptibility curves as a function of temperature for Eu-free $\mathrm{Ba}_{8} \mathrm{Al}_{5} \mathrm{Si}_{41}$ in applied fields from 100 to $5 \mathrm{kOe}$. With applied field increasing, the suppression in $T_{\mathrm{C}}$ is small and not clearly visible in this figure. However, we observed a strong reduction in superconducting volume $V_{\text {sup }}$. As shown in the inset in Fig. 3, $V_{\text {sup }}$ decreases with increasing field in a $\log \operatorname{linear}$ behavior, $\log V_{\text {sup }}=a+b \log H$, with $a=4.1$ and $b=-1.5$.

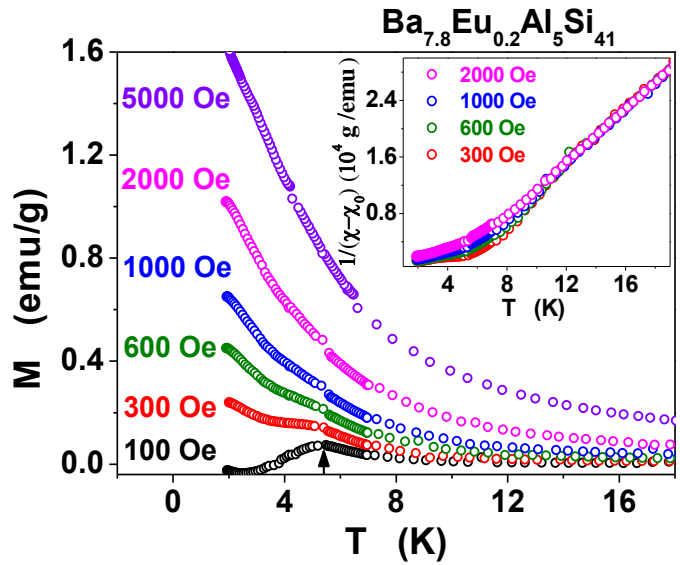

Fig. 4. The ZFC magnetization versus temperature for $\mathrm{Ba}_{7.8} \mathrm{Eu}_{0.2} \mathrm{Al}_{5} \mathrm{Si}_{41}$ under fields from 100 to 5000 Oe. Inset: inverse susceptibility with the same curves.

The temperature dependence of the magnetization for specimen $\mathrm{Ba}_{7.8} \mathrm{Eu}_{0.2} \mathrm{Al}_{5} \mathrm{Si}_{41}$ under fields from 100 to $5 \mathrm{kOe}$ is shown in Fig. 4. The superconducting diamagnetic transition occurs at $5.2 \mathrm{~K}$ as denoted by the dark arrow. With the field increasing, the superconducting diamagnetic signal is 
weakened and then disappears. It is clear that the magnetic moment of Eu-doped sample is strengthened as a result of the increasing of the field, so the superconductivity was strongly depressed until vanished.

For the $\mathrm{Ba}_{7.8} \mathrm{Eu}_{0.2} \mathrm{Al}_{5} \mathrm{Si}_{41}$ sample, the Curie paramagnetic behavior appears at high temperature due to the incorporation of magnetic Eu ions into the clathrate cages. A Curie-Weiss fit of susceptibility $\chi=C /\left(T-\theta_{w}\right)+\chi_{\mathrm{o}}\left(\chi_{\mathrm{o}}\right.$ is temperature-independent magnetic background, $C$ is the material-specific Curie constant) for high temperature data under 600 Oe yielded the Weiss temperature $\theta_{w} \sim 5.57 \mathrm{~K}$. The effective magnetic moment calculated according to the fitted $C$ to the data under 2000 Oe was $7.45 \mu_{\mathrm{B}}$, this value is about $6 \%$ smaller than that the free $\mathrm{Eu}^{2+}$ ion value $\left(7.93 \mu_{\mathrm{B}}\right)$. The inverse magnetic susceptibility $1 /\left(\chi-\chi_{\mathrm{o}}\right)$ vs temperature curves in fields of 300 , 600,1000 and 2000 Oe were plotted in the inset. The curves are straight line above approximately $8 \mathrm{~K}$, confirming the Curie paramagnetic behavior at high temperature. The positive intercept on the $T$ axis from the extrapolation of the straight line implies the onset of ferromagnetic interaction.

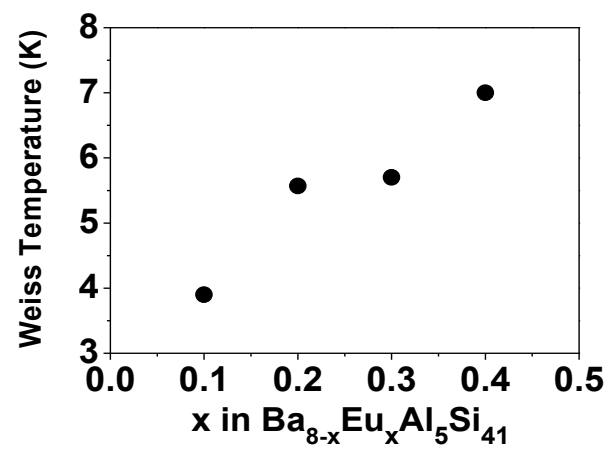

Fig. 5. The Weiss temperature $\theta_{w}$ obtained from the Curie-Weiss fits to the $\chi-T$ curves at high temperature taken under 600 Oe for samples $\mathrm{Ba}_{8-x} \mathrm{Eu}_{x} \mathrm{Al}_{5} \mathrm{Si}_{41}(x=0.1$, $0.2,0.3$ and 0.4$)$.

With the increase of the doped Eu content in the clathrate, the averaged Eu-Eu distance decreases and the interaction between them is enhanced. As a result, the superconducting transition is depressed and Weiss temperature increases. For samples $\mathrm{Ba}_{8-x} \mathrm{Eu}_{x} \mathrm{Al}_{5} \mathrm{Si}_{41}$ with $x=$ $0.1,0.2,0.3$ and 0.4, Fig. 5 gives the Weiss temperature $\theta_{w}$ yielded from the Curie-Weiss fits to the $\chi$-T curves taken under field $600 \mathrm{Oe}$. As can be seen in the figure 5, the Weiss temperature increases with the increase of Eu content. It should be pointed out that there is little difference between the $\theta_{w}$ of samples $x=0.2$ and 0.3 . This must be related to the approximate Eu content (see table 1) of them. 


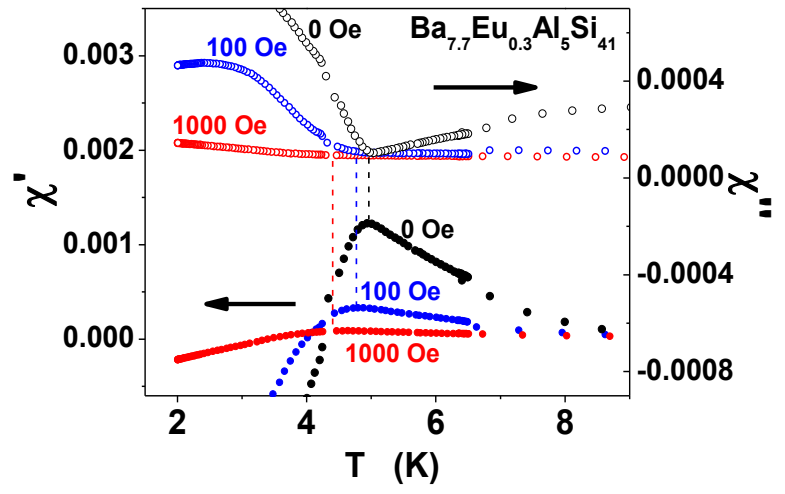

Fig. 6. Temperature variation of the ac magnetic susceptibility under different magnetic fields for sample $\mathrm{Ba}_{7.7} \mathrm{Eu}_{0.3} \mathrm{Al}_{5} \mathrm{Si}_{41}$.

The temperature dependence of the ac susceptibility for sample $\mathrm{Ba}_{7.7} \mathrm{Eu}_{0.3} \mathrm{Al}_{5} \mathrm{Si}_{41}$ was measured under 0, 100, and 1000 Oe static field, respectively, with ac field amplitude of 3 Oe at a frequency of $256 \mathrm{~Hz}$. The results are shown in Fig. 6. With the decrease of the temperature, the sample turns into the superconducting state. The in-phase susceptibility $\chi^{\prime}$ drops and the out-of-phase susceptibility $\chi^{\prime \prime}$ peaks at the same time. The transition temperature decreases form 4.9 to $4.3 \mathrm{~K}$ as the static magnetic field increases from 0 to $1000 \mathrm{Oe}$.

3.3. Magnetization ( $M-H)$ curves

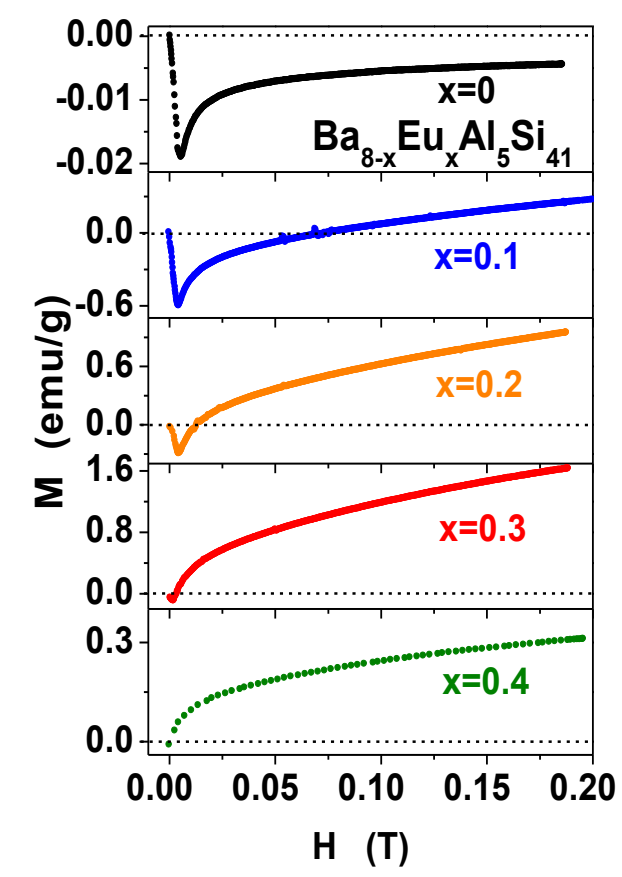

Fig. 7. Initial magnetization curves at $2 \mathrm{~K}$ for samples $\mathrm{Ba}_{8-x} \mathrm{Eu}_{x} \mathrm{Al}_{5} \mathrm{Si}_{41}(x=0$, $0.1,0.2,0.3$ and 0.4$)$.

The $M-H$ curves at $2 \mathrm{~K}$ for all samples at low field $(0-0.2 \mathrm{~T})$ were plotted in Fig. 7. The black dashed line is the horizontal line of zero magnetization. For Eu-free sample $(x=0)$, the trend of $M$ with increasing $H$ is the characteristic initial magnetization behavior of type-II 
superconductor. At small fields, shieling is $100 \%$ and $|M|$ versus $H$ is approximately linear. With the increase of the magnetic field, flux penetrates in the superconductor in the form of vortices. The critical field where the $|M|$ deviates from the linearity is defined as the lower critical field $H_{\mathrm{C} 1}$. At high field, $|M|$ gradually decreases and closes to zero.

For the Eu-doped samples, the superconductivity was suppressed and the ferromagnetic behavior was induced at the same time due to the introduction of the magnetic moment of $\mathrm{Eu}^{2+}$. Therefore, the $H_{\mathrm{C} 1}$ decreases from 50 to $16.8 \mathrm{Oe}$ as $x$ increases from 0 to 0.3 . Besides, the saturation magnetization of Eu-doped samples is greater than zero and increases rather strikingly with $x$. As $x$ reaches to 0.4 , no any diamagnetization line can be observed at lower fields due to the disappearance of superconductivity. The curve shows a very sharp increase in magnetization, as expected in pure ferromagnetic materials.

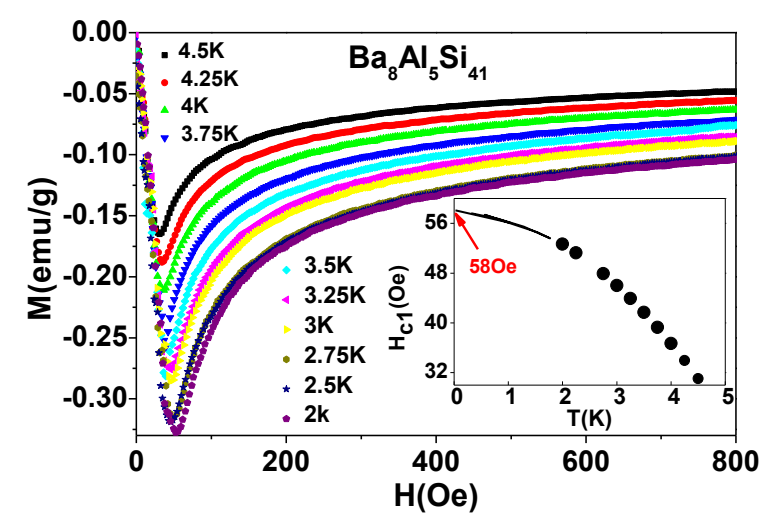

Fig. 8. The magnetization curves at various temperatures from 2 to $4.5 \mathrm{~K}$ for Eu-free $\mathrm{Ba}_{8} \mathrm{Al}_{5} \mathrm{Si}_{41}$ sample. The inset shows $T$ dependence of lower critical field $H_{\mathrm{C} 1}$. The solid line is a fit assuming a parabolic $T$ dependence, giving $H_{\mathrm{Cl}}(0)=58$ Oe.

For the Eu-free $\mathrm{Ba}_{8} \mathrm{Al}_{5} \mathrm{Si}_{41}$, the magnetization curves at various temperatures below $T_{\mathrm{C}}$ are plotted in Fig. 8. The $H_{\mathrm{Cl}}$ decreases with temperature, as shown in the inset. The solid line represents a fit to the data using a simple parabolic $T$ dependence in the form of $H_{\mathrm{C} 1}(T)=H_{\mathrm{C} 1}(0)\left(1-t^{2}\right)$, where $t=T / T_{\mathrm{C}}$. The extrapolated $H_{\mathrm{C} 1}$ at $0 \mathrm{~K}$ is about $58 \mathrm{Oe}$, which is higher than the 40.2 Oe obtained for the $\mathrm{Ba}_{8} \mathrm{Al}_{4} \mathrm{Si}_{42}$ sample reported in ref [24].
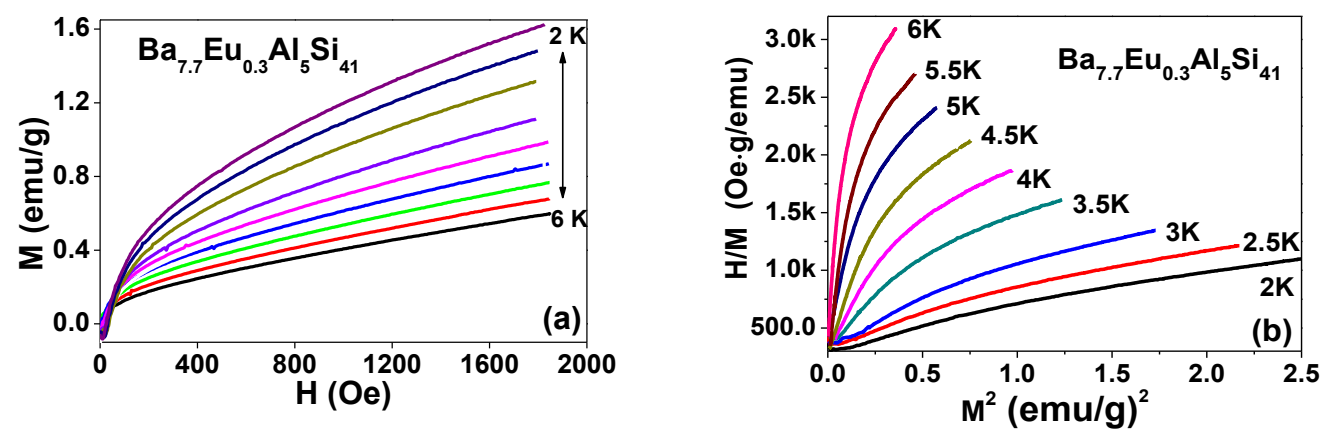

Fig. 9. Magnetization isotherms (a) and the $H / M$ vs. $M^{2}$ plots (b) measured at different temperatures between 2 and $6 \mathrm{~K}$ with $0.5 \mathrm{~K}$ interval for nominal $\mathrm{Ba}_{7.7} \mathrm{Eu}_{0.3} \mathrm{Al}_{5} \mathrm{Si}_{41}$ sample. 
Fig. 9 (a) shows a series of $M-H$ isotherms taken at $0.5 \mathrm{~K}$ intervals from 2 to $6 \mathrm{~K}$ for nominal $\mathrm{Ba}_{7.7} \mathrm{Eu}_{0.3} \mathrm{Al}_{5} \mathrm{Si}_{41}$ sample. Below $T_{\mathrm{C}}(\sim 4.5 \mathrm{~K})$, the superconductivity is in coexistence with the ferromagnetism behavior. Sample shows the superconducting diamagnetic behavior below the $H_{\mathrm{C} 1}$ ( 17 Oe). Above $T_{\mathrm{C}}$, no superconducting diamagnetic can be observed and magnetization increases monotonously with temperature. The corresponding $H / M$ versus $M^{2}$ plots (the so-called Arrott plots) are shown in Fig. 9 (b). The presence of the positive slope of the $H / M$ versus $M^{2}$ curves indicates that the magnetic transition is of second order. This result is consistent with the absence of thermal hysteresis of $M-T$ curves at high fields.

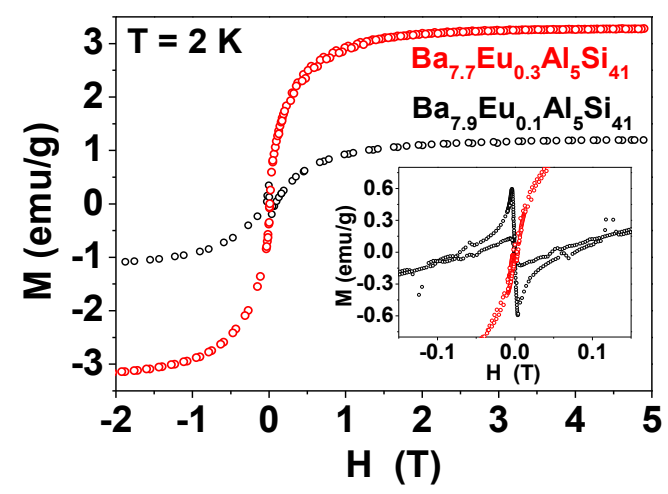

Fig. 10. $M$ - $H$ hysteresis of nominal $\mathrm{Ba}_{7.9} \mathrm{Eu}_{0.1} \mathrm{Al}_{5} \mathrm{Si}_{41}$ and $\mathrm{Ba}_{7.7} \mathrm{Eu}_{0.3} \mathrm{Al}_{5} \mathrm{Si}_{41}$ at $2 \mathrm{~K}$.

The hysteresis loops at $2 \mathrm{~K}$ were measured for nominal $\mathrm{Ba}_{7.9} \mathrm{Eu}_{0.1} \mathrm{Al}_{5} \mathrm{Si}_{41}$ and $\mathrm{Ba}_{7.7} \mathrm{Eu}_{0.3} \mathrm{Al}_{5} \mathrm{Si}_{41}$. As seen in Fig. 10, the superconductivity and ferromagnetism are coexistence. The inset shows the enlarged $M-H$ curves between -0.15 and $0.15 \mathrm{~T}$. The negative $M$ is approximately linear with $H$, originates from the diamagnetism below the superconducting lower critical field $H_{\mathrm{Cl}}$. Then $M$ becomes positive and displays a sharp increase with field, as ordinary seen in ferromagnetic materials. The saturation moment per Eu atom reaches $\sim 5.1$ and $4.7 \mu_{\mathrm{B}}$, the value of which was estimated by extrapolation to the zero magnetic filed from the linear portion of the curve. The coercive force is very small and the field hysteresis is negligible, means a very soft magnetic behavior.

\section{Conclusion}

The silicon clathrate superconductor is uncommon as its structure is dominated by strong $\mathrm{Si}-\mathrm{Si}$ covalent bonds, rather than the metallic bond that are more typical of traditional superconductors. To understand the influence of large magnetic moment of Eu on properties of type-I clathrates, a series of dilute Eu-doped samples with the nominal formula $\mathrm{Ba}_{8-x} \mathrm{Eu}_{x} \mathrm{Al}_{5} \mathrm{Si}_{41}$ ( $x$ $=0,0.1,0.2,0.3$ and 0.4) were synthesised in which Eu occupied Ba sites in cage center. The structural and chemical characterizations, magnetic heat ( $M-T)$ and magnetization $(M-H)$ curves were measured for all samples. Eu atoms enter the clathrate lattice and their magnetic moments break paired electrons so superconductivity was depressed. On the other hand, the substitution of $\mathrm{Eu}$ for Ba results in a ferromagnetic behavior at low temperature and the Curie-Weiss temperature 
increases with the increase of the Eu content.

\section{Acknowledgments}

This work is supported by "the Fundamental Research Funds for the Central Universities" (Grant No. FRF-BR-15-007A). YL was supported in part by the National Science Foundation (DMR-0821284 and 1531755), NASA (NNX10AM80H and NNX07AO30A).

\section{References}

[1] E. S. Toberer, A. F. May, G. J. Snyder, Chem. Mater. 22 (2010) 624-634.

[2] J. R. Sootsman, D. Y. Chung, M. G. Kanatzidis, Angew. Chem. Int. Ed. 48 (2009) 8616-8639.

[3] G. J. Snyder, E. S. Toberer, Nat. Mater. 7 (2008) 105-114.

[4] A. Saramat, G. Svensson, A. E. C. Palmqvist, C. Stiewe, E. Mueller, D. Platzek, S. G. K. Williams, D. M. Rowe, J. D. Bryan, G. D. Stucky, J. Appl. Phys. 99 (2006) 023708.

[5] Y. Li, J. Garcia, N. Chen, L. Liu, F. Li, J. Appl. Phys. 113 (2013) 203908.

[6] Y. Li, R. Zhang, Y. Liu, N. Chen, Z. P. Luo, X. Ma, G. Cao, Z. S. Feng, Chia-Ren Hu, J. H. Ross, Jr., Phys. Rev. B 75 (2007) 054513.

[7] S. Yamanaka, E. Enishi, H. Fukuoka, M. Yasukawa, Inorg. Chem. 39 (2000) 56-58.

[8] K. Tanigaki, T. Shimizu, K. M. Itoh, J. Teraoka, Y. Moritomo, S. Yamanaka, Nat. Mater. 2 (2003) 653-655.

[9] G. B. Adams, M. O'Keefe, A. A. Demkov, O. F. Sankey, Yin-Min. Huang, Phys. Rev. B 49, (1994) 8048.

[10] A. San-Miguel, P. Kéghélian, X. Blase, P. Mélinon, A. Perea, J. P. Itié, A. Polian, E. Reny, C. Cros, M. Pouchard, Phys. Rev. Lett. 83 (1999) 5290-5293.

[11] A. San-Miguel, P. Toulemonde, High Pressure Res. 25 (2005) 159-185.

[12] J. H. Ross Jr., Y. Li, Superconductivity and magnetism in silicon and germanium clathrates, in: Nanoscale Magnetic Materials and Applications, Springer, 2009, pp. 105 - 122.

[13] L. Liu, S. Bi, N. Chen, F. Li, Y. Liu, G. Cao, Y. Li, Physica C 506 (2014) 94-99.

[14] L. Liu, S. Bi, B. Peng, Y. Li, J. Appl. Phys. 117 (2015) $17 E 117$.

[15] L. Liu, B. Song, B. Sun, H. Ma, X. Ma, Y. Li, Physica B 485 (2016) 84-88.

[16] W. Jung, H. Kessens, A. Ormeci, W. Schnelle, U. Burkhardt, H. Borrmann, H. D. Nguyen, M. Baitinger, Y. Grin, Dalton Trans. 41 (2012) 13960-13968.

[17] B. C. Sales, B. C. Chakoumakos, R. Jin, J. R. Thompson, D. Mandrus, Phys. Rev. B 63 (2001) 245113.

[18] Y. Mudryk, P. Rogl, C. Paul, S. Berger, E. Bauer, G. Hilscher, C. Godart, H. Noël, J. Phys.: Condens. Matter 14 (2002) 7991-8004.

[19] L. M. Holanda, J. M. Vargas, C. Rettori, P. G. Pagliuso, E. M. Bittar, M. A. Avila, T. Takabatake, Physica B 404 (2009) 3300-3303.

[20] P. Toulemonde, Ch. Adessi, X. Blase, A. San Miguel, Phys. Rev. B 71 (2005) 094504.

[21] S. Johnsen, A. Bentien, G. K. H. Madsen, M. Nygren, B. B. Iversen, Phys. Rev. B 76 (2007) 245126.

[22] S. Johnsen, A. Bentien, G. K. H. Madsen, B. B. Iversen, Chem. Mater. 18 (2006) 4633-4642.

[23] M. Hokazono, H. Anno, K. Matsubara, Mater. Trans. 46 (2005) 1485-1489.

[24] Y. Li, J. Garcia, G. Franco, J. Lu, K. Lu, B. Rong, B. Shafiq, N. Chen, Y. Liu, L. Liu, B. Song, 
Y. Wei, S. S. Johnson, Z. Luo, Z. Feng, J. Appl. Phys. 117 (2015) 213912. 
Nominal composition

$\mathrm{Ba}_{8} \mathrm{Al}_{5} \mathrm{Si}_{41}$

$\mathrm{Ba}_{7.9} \mathrm{Eu}_{0.1} \mathrm{Al}_{5} \mathrm{Si}_{41}$

$\mathrm{Ba}_{7.8} \mathrm{Eu}_{0.2} \mathrm{Al}_{5} \mathrm{Si}_{41}$

$\mathrm{Ba}_{7.7} \mathrm{Eu}_{0.3} \mathrm{Al}_{5} \mathrm{Si}_{41}$

$\mathrm{Ba}_{7.6} \mathrm{Eu}_{0.4} \mathrm{Al}_{5} \mathrm{Si}_{41}$
Composition by refinement

$$
\mathrm{Ba}_{7.81} \mathrm{Al}_{6.44} \mathrm{Si}_{39.53}
$$

$\mathrm{Ba}_{7.63} \mathrm{Eu}_{0.08} \mathrm{Al}_{6.00} \mathrm{Si}_{39.83}$

$\mathrm{Ba}_{7.69} \mathrm{Eu}_{0.16} \mathrm{Al}_{5.95} \mathrm{Si}_{40.05}$

$\mathrm{Ba}_{7.61} \mathrm{Eu}_{0.24} \mathrm{Al}_{5.88} \mathrm{Si}_{40.01}$

$\mathrm{Ba}_{7.49} \mathrm{Eu}_{0.35} \mathrm{Al}_{5.89} \mathrm{Si}_{40.09}$
Composition by EDS

$$
\mathrm{Ba}_{8.00} \mathrm{Al}_{6.31} \mathrm{Si}_{39.69}
$$

$\mathrm{Ba}_{7.91} \mathrm{Eu}_{0.09} \mathrm{Al}_{6.21} \mathrm{Si}_{39.79}$

$\mathrm{Ba}_{7.77} \mathrm{Eu}_{0.23} \mathrm{Al}_{5.87} \mathrm{Si}_{40.13}$

$\mathrm{Ba}_{7.75} \mathrm{Eu}_{0.25} \mathrm{Al}_{5.45} \mathrm{Si}_{40.55}$

$\mathrm{Ba}_{7.63} \mathrm{Eu}_{0.37} \mathrm{Al}_{5.59} \mathrm{Si}_{40.41}$ 


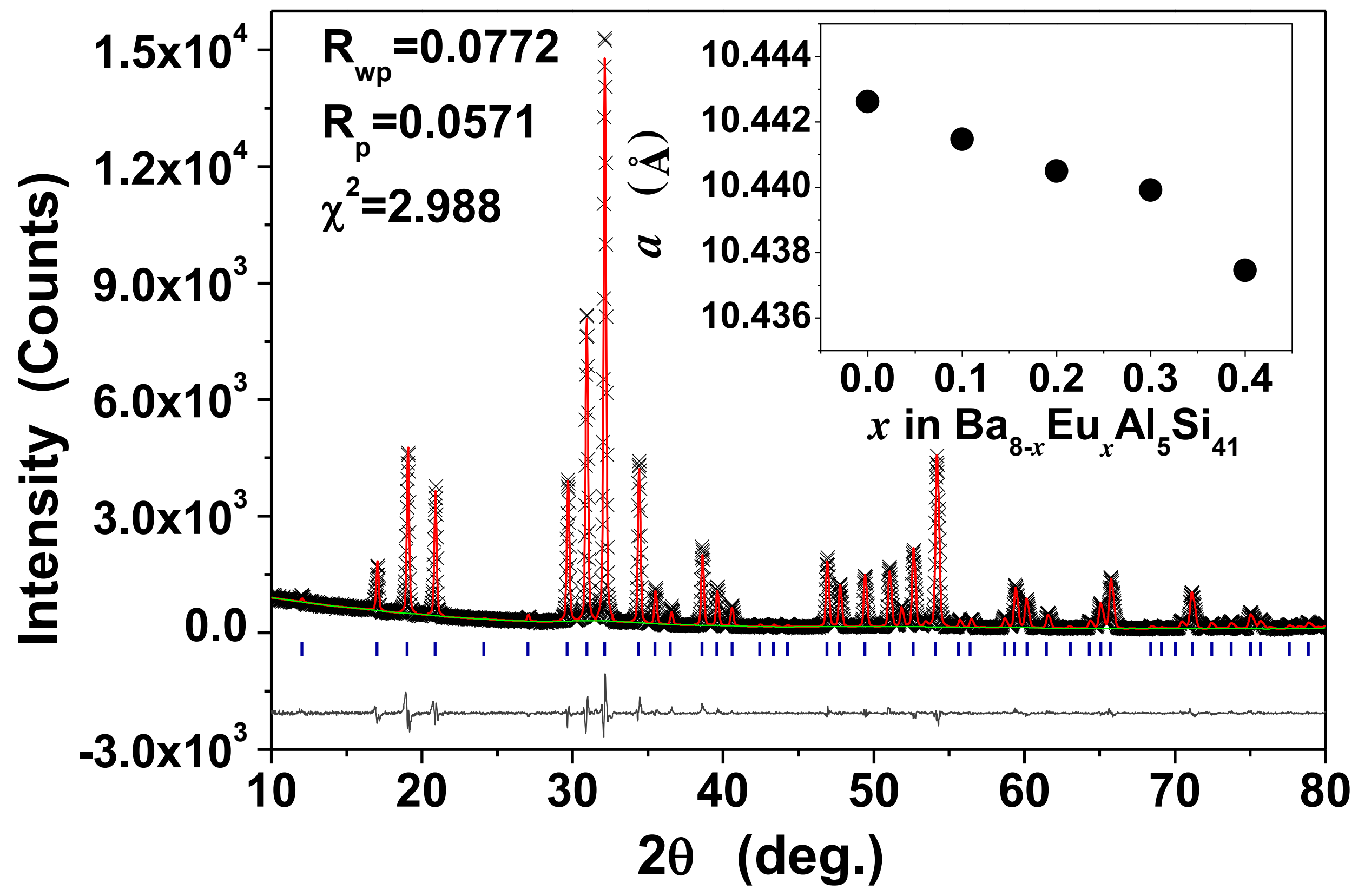




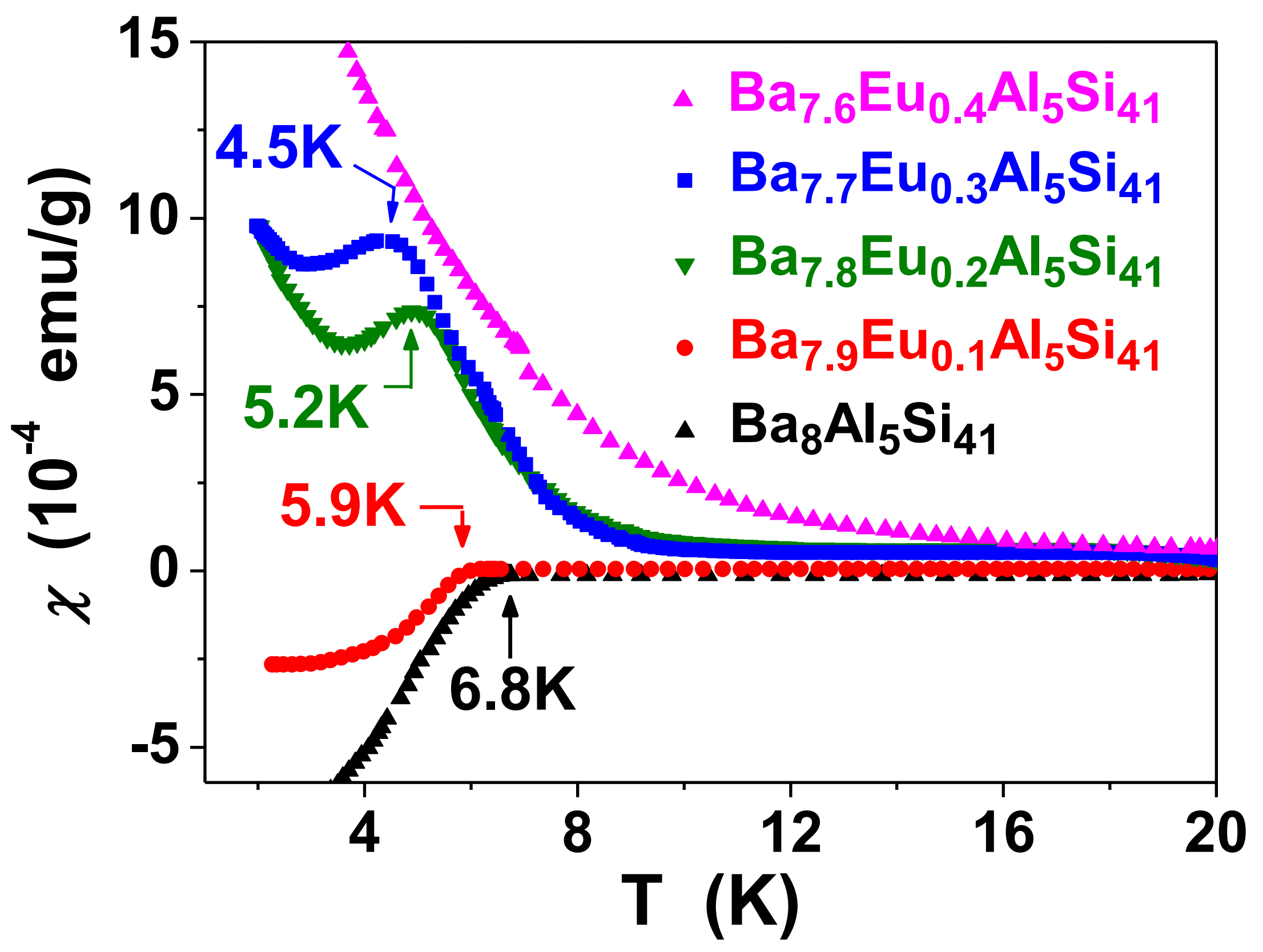




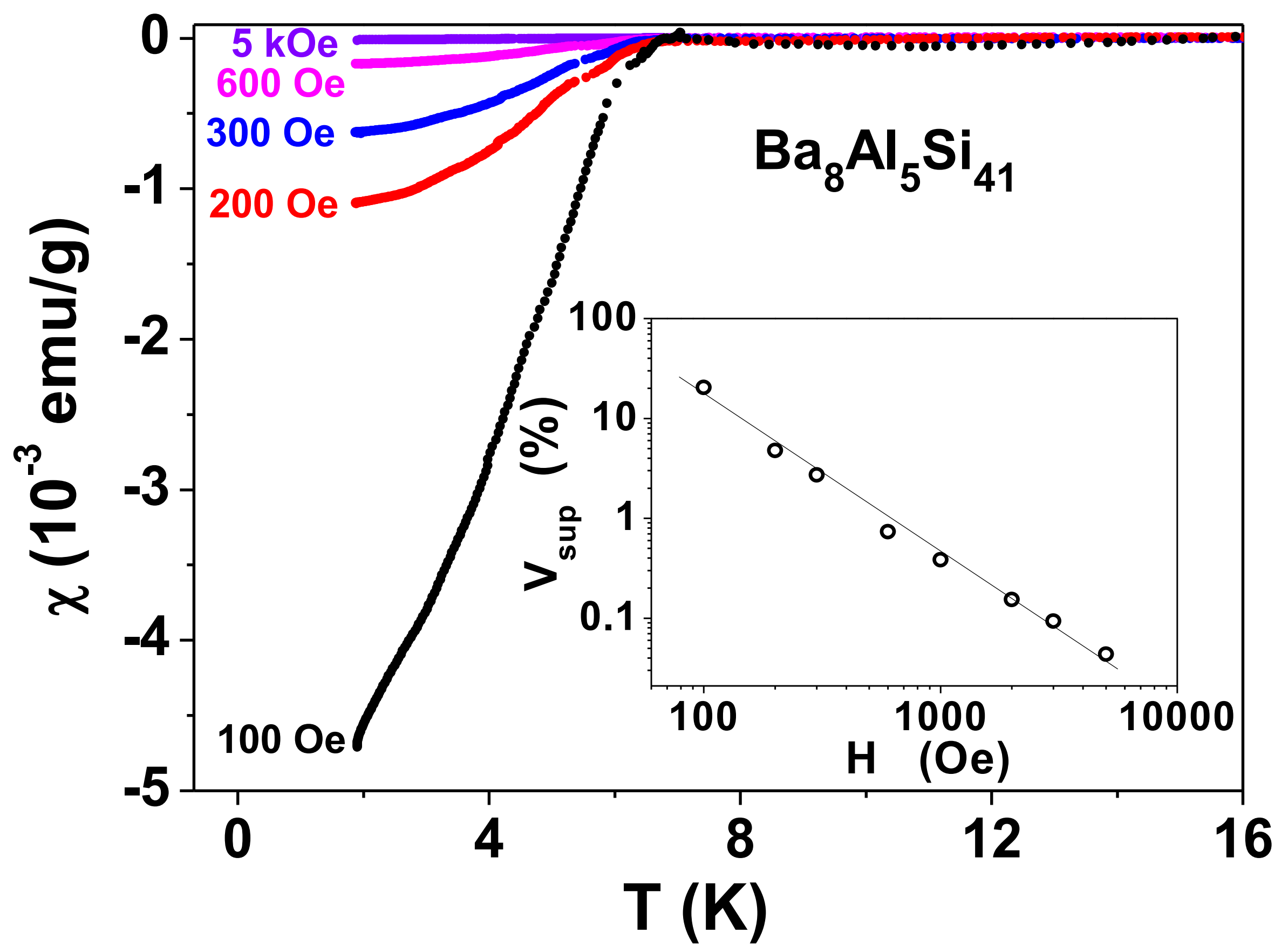




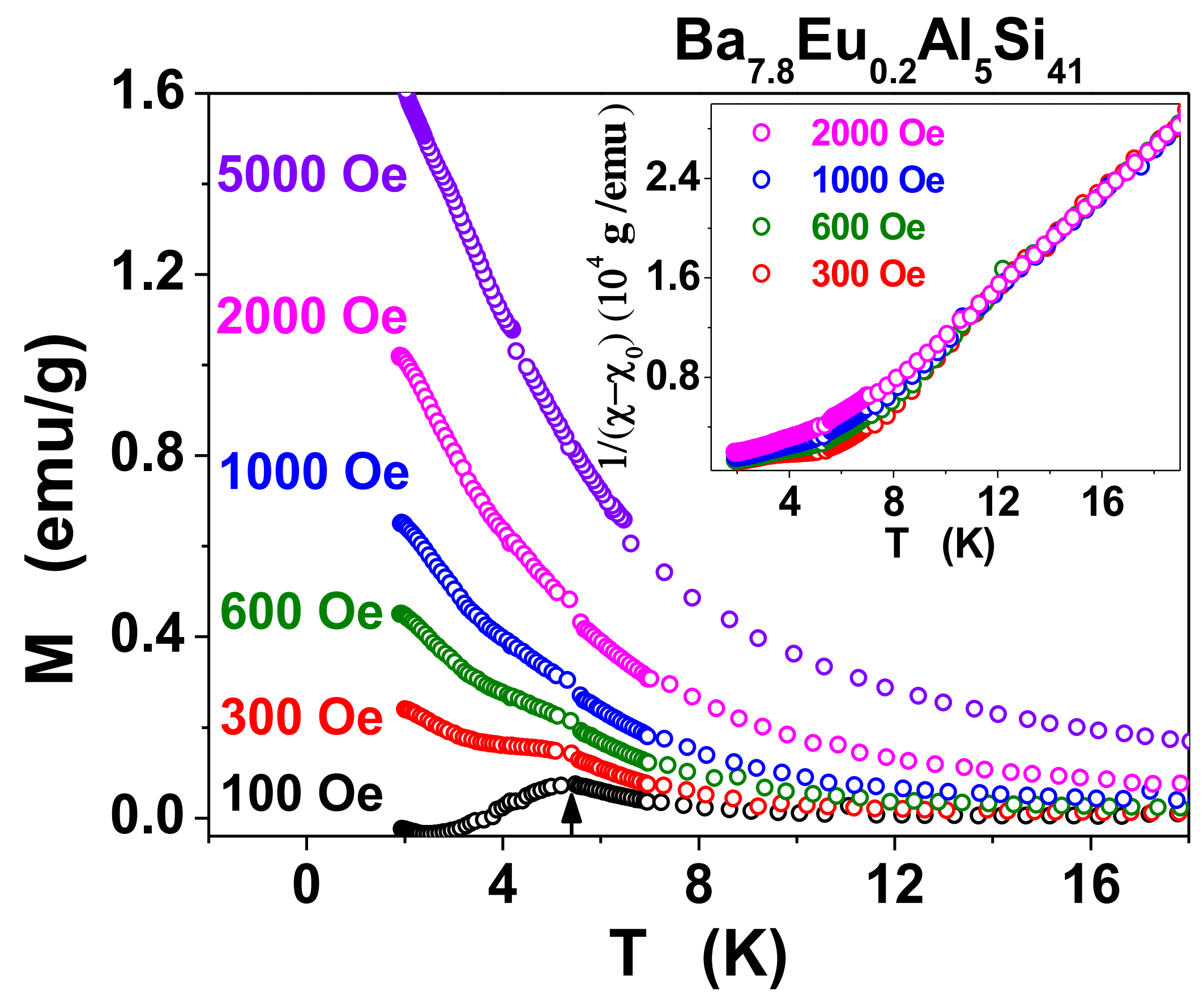




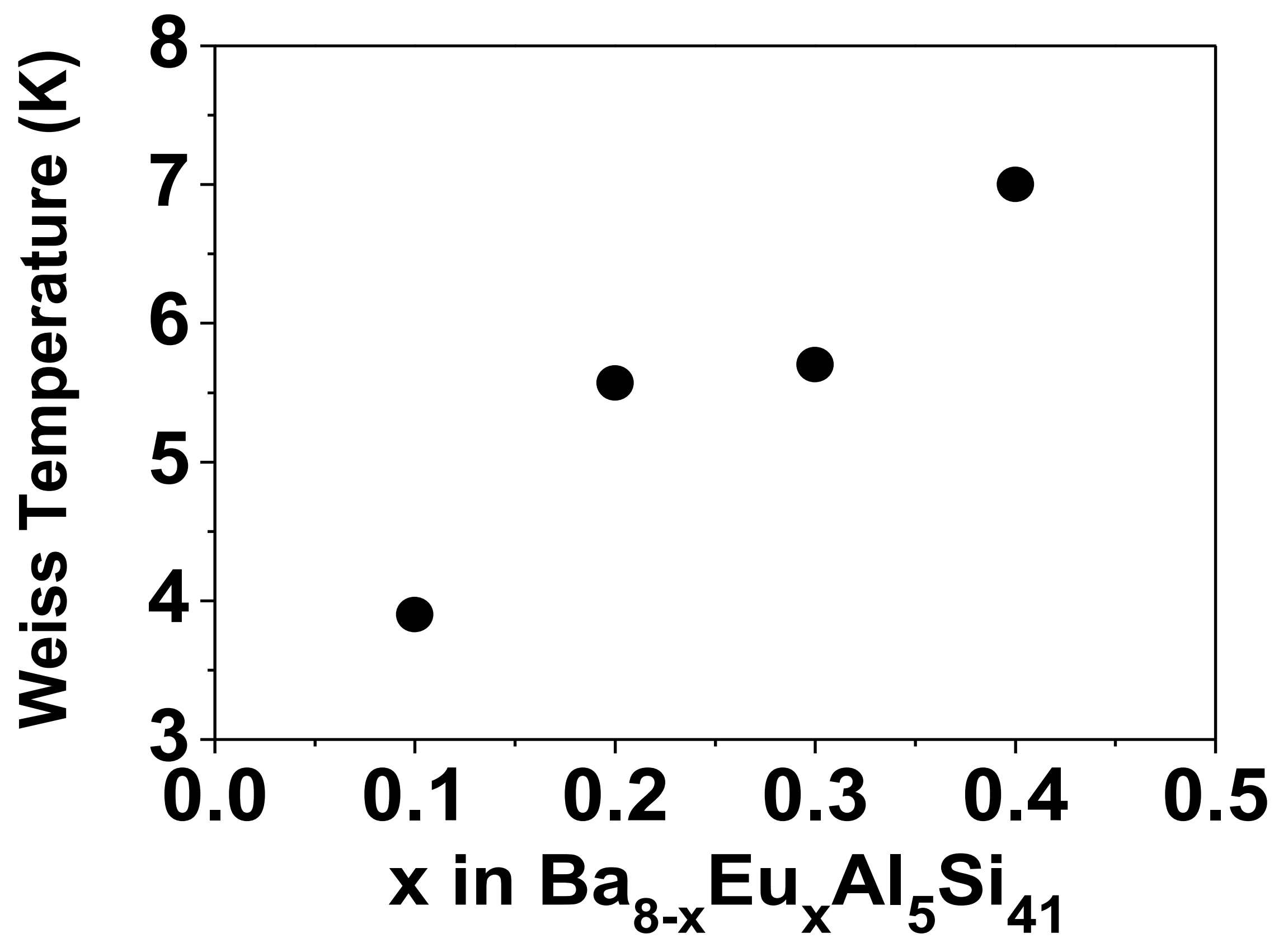




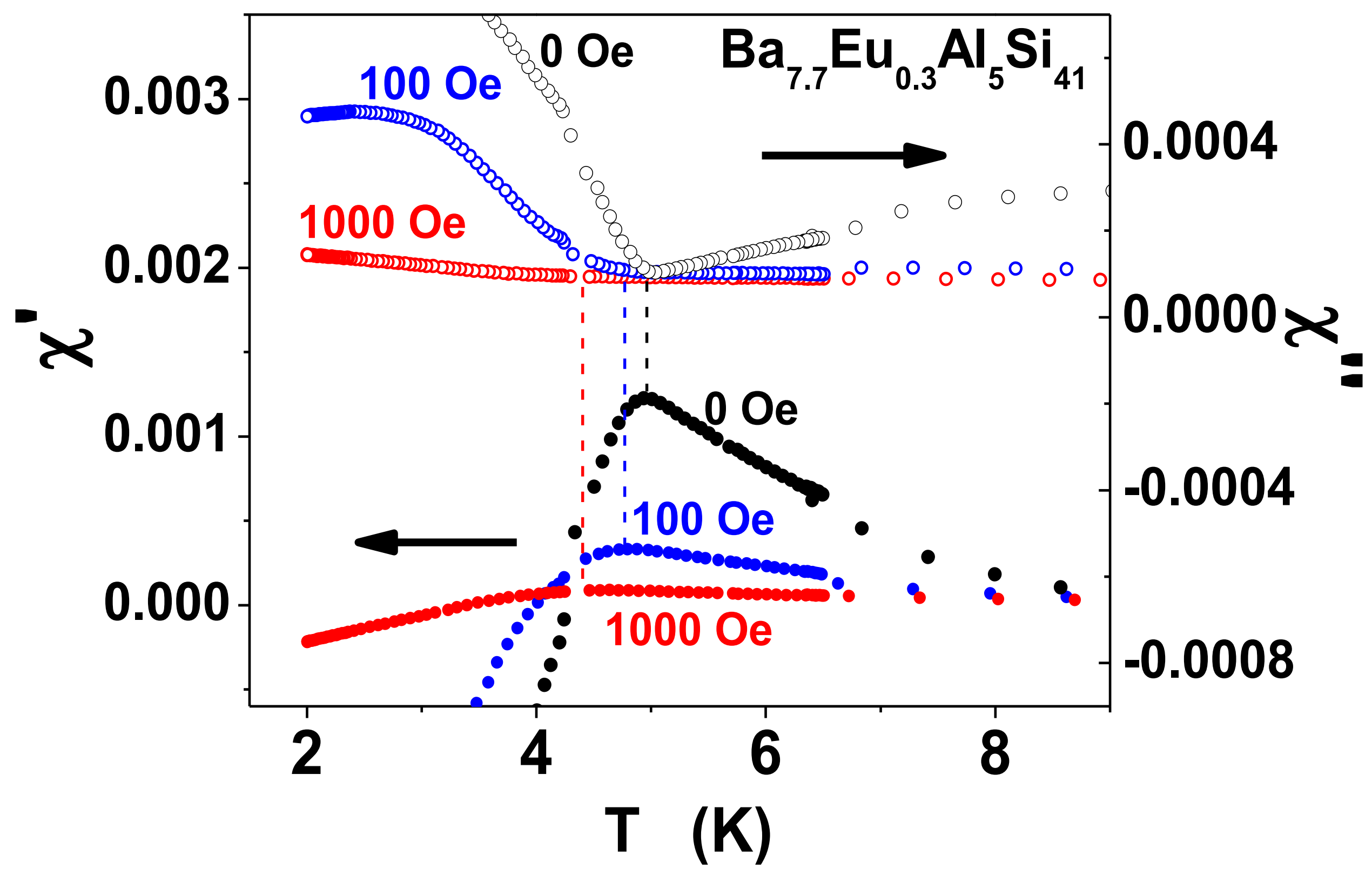




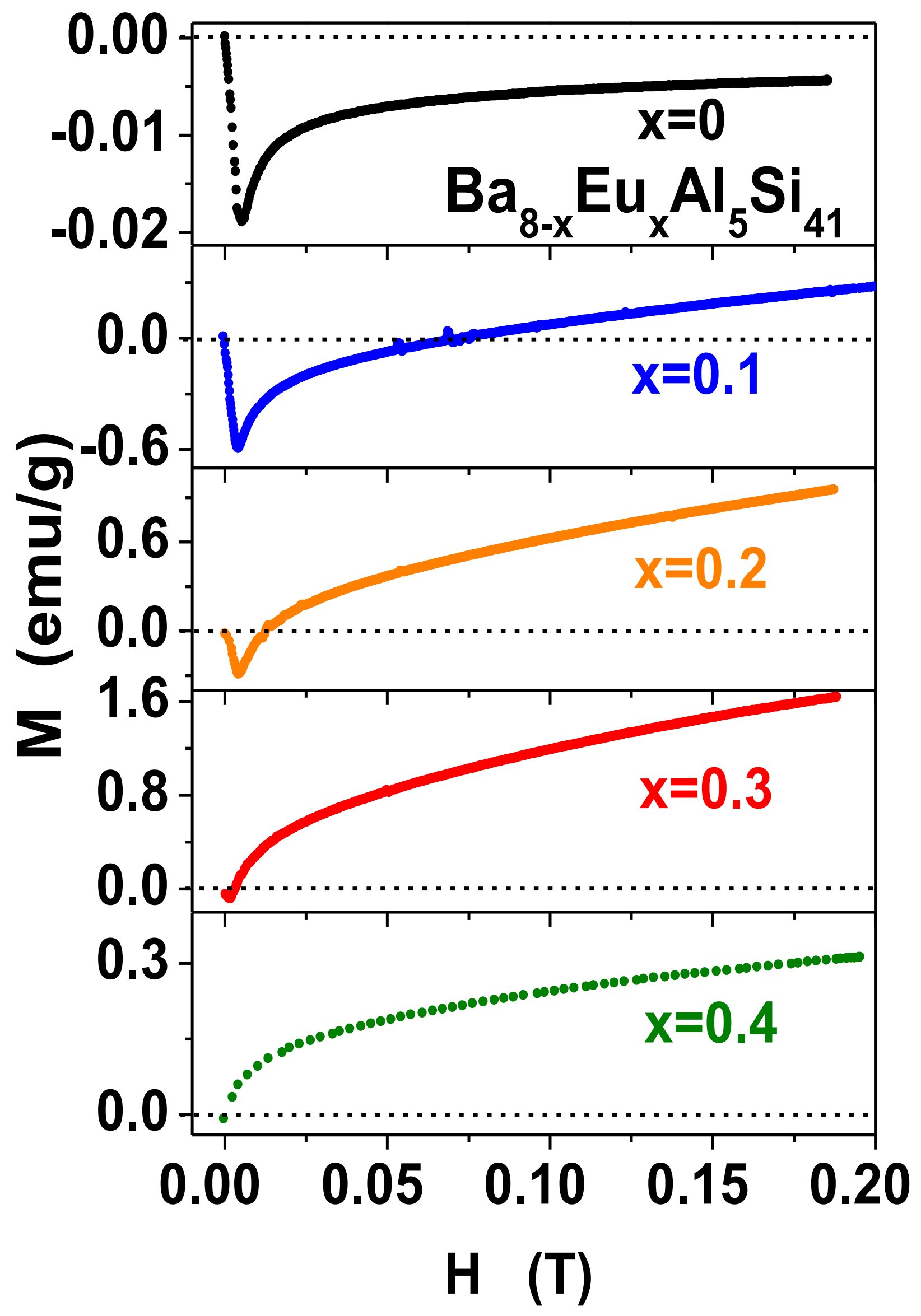




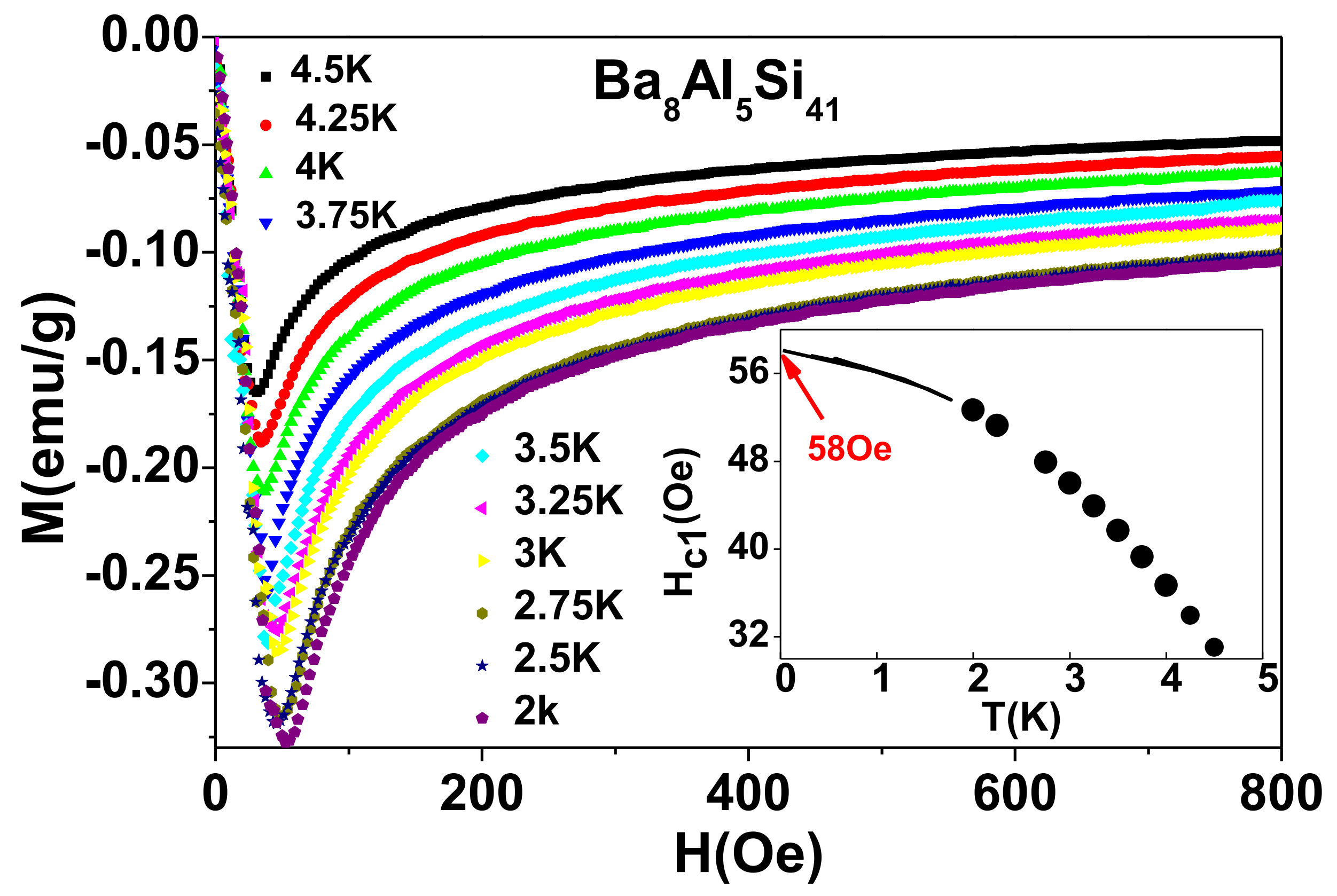



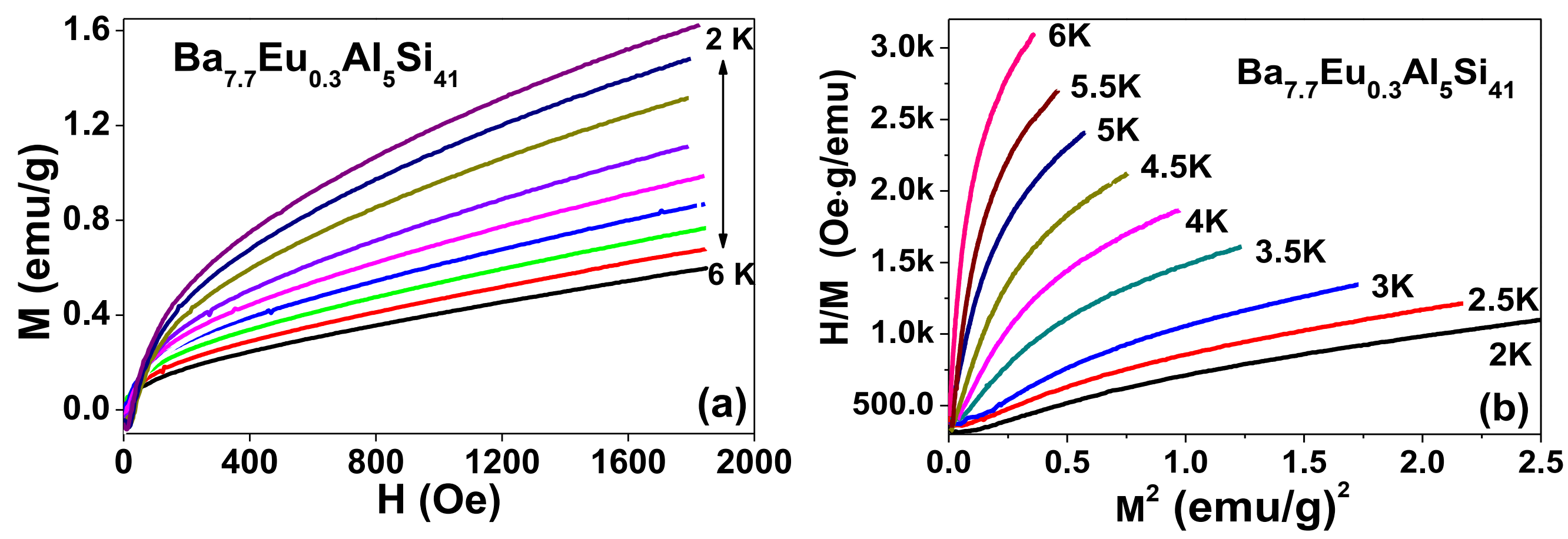


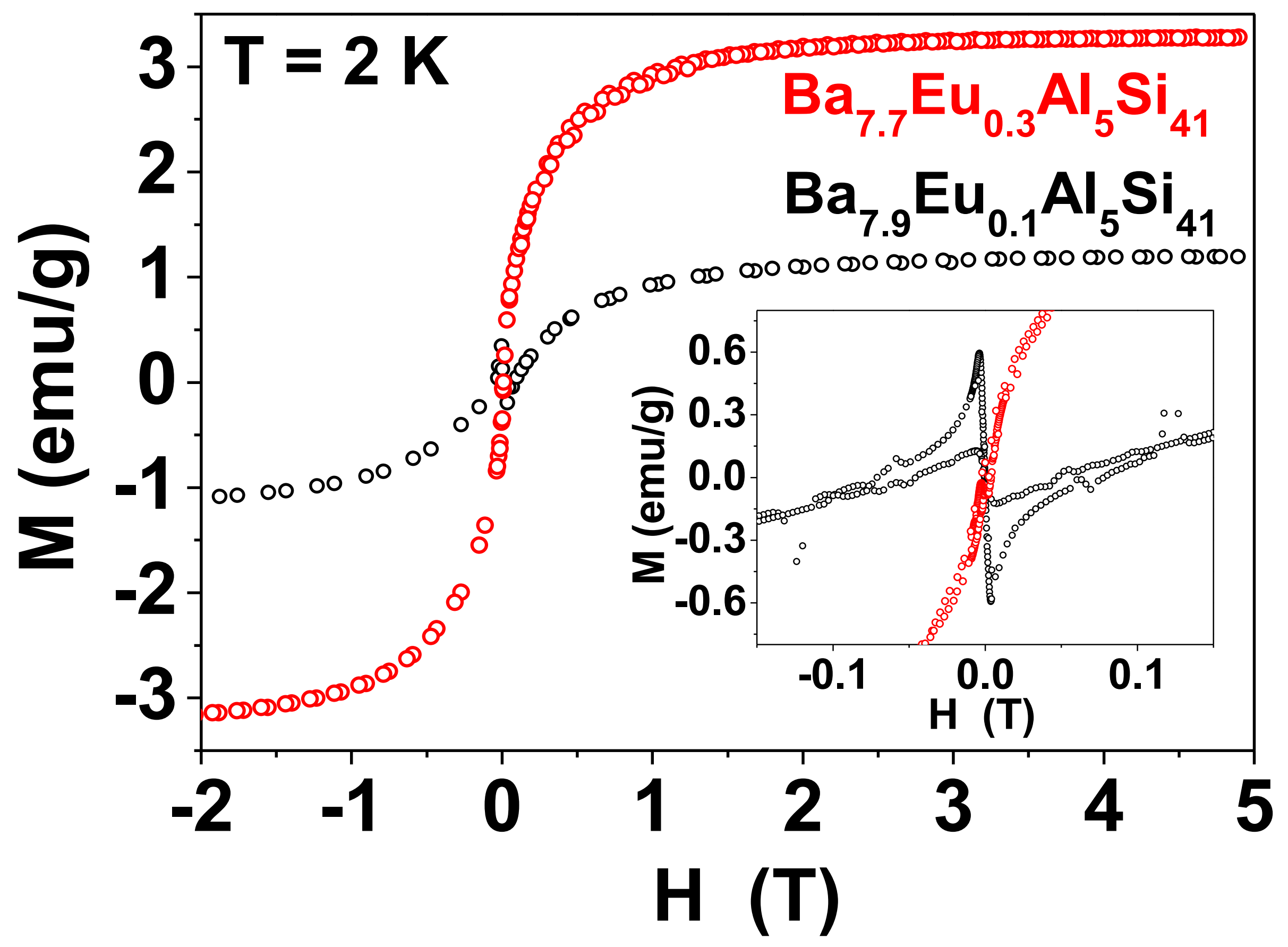

